\title{
Successful endoluminal rescue of an endovascular graft unintentionally deployed in the false lumen of Stanford type B aortic dissection
}

\author{
Xiang Ma, MD, PhD, ${ }^{\text {a }}$ Weiguo Fu, MD, PhD, ${ }^{\mathrm{b}}$ Yi-Tong Ma, MD, PhD, ${ }^{\mathrm{a}}$ Ujit Karmacharya, MD, ${ }^{\mathrm{a}}$ and \\ Jun Zhao, $\mathrm{MD}, \mathrm{PhD},{ }^{\mathrm{c}}$ Xinjiang and Shanghai, China
}

\footnotetext{
From the a Department of Cardiology, The First Affiliated Hospital of Xinjiang Medical University, Xinjiang; ${ }^{\mathrm{b}}$ Department of Vascular Surgery, Zhongshan Affiliated Hospital of Shanghai Fudan University; ${ }^{\mathrm{c}}$ Division of Vascular Surgery, Department of General Surgery, The Sixth Affiliated People's Hospital of Shanghai Jiao Tong University, Shanghai, China.

Disclosures: Authors have nothing to disclose with regard to commercial support.

X.M. and W.F. contributed equally to this work.

Received for publication June 8, 2015; revisions received Oct 15, 2015; accepted for publication Oct 22, 2015; available ahead of print Dec 2, 2015

Address for reprints: Jun Zhao, MD, PhD, Division of Vascular Surgery, Department of General Surgery, The Sixth Affiliated People's Hospital of Shanghai Jiao Tong University, Shanghai, China 200233 (E-mail: zhao_jun@yahoo.com).

J Thorac Cardiovasc Surg 2016;151:e41-5

$0022-5223 / \$ 36.00$

Copyright (c) 2016 by The American Association for Thoracic Surgery

http://dx.doi.org/10.1016/j.jtcvs.2015.10.083
}

Thoracic endovascular aortic repair (TEVAR) has been used increasingly as a less-invasive alternative to open surgery for patients with Stanford type B aortic dissection. However, a stent graft erroneously deployed into the false lumen may cause a rare and devastating complication. It increases the risk of early rupture and necessitates an emergency rescue procedure. We report a case that was rescued by a fenestration procedure, involving a tubular stent graft "homing technique" to draw the blood flow back into the true lumen. In addition, a large distal reentry tear was sealed with coils plus bare stents. The details are presented as follows.

\section{CASE PRESENTATION}

A 42-year-old hypertensive, nondiabetic, nonsmoker, and nonalcoholic man suddenly felt chest pain and palpitations while doing his household chores. On arrival to the emergency department, his vital signs were stable, except he had a blood pressure of 180/90 mm Hg. Electrocardiogram showed sinus rhythm. Echocardiography showed evidence of Stanford type B thoracic aortic dissection.

Contrast computed tomography (CT) showed Stanford type B aortic dissection with periaortic hematoma, hemorrhagic pleural effusion, and atelectatic lungs. Ultrasoundguided $10 \mathrm{~mL}$ drainage of blood from the effusion site confirmed the CT findings as complicated type B dissection. The main entry tear was located in the descending aorta $20 \mathrm{~mm}$ away from the ostium of the left subclavian artery (LSA).

After management of hypertension, the patient was prepared for TEVAR. The procedure was performed under general anesthesia with full hemodynamic monitoring. Two pigtail catheters were positioned, one in the ascending the CT findings. pain.

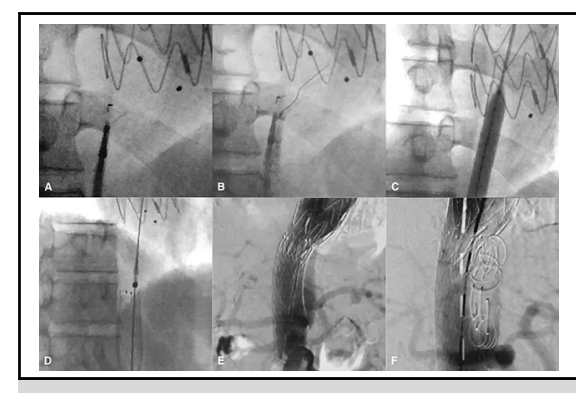

Successful endoluminal treatment of endovascular graft deployed in the false lumen.

\section{Central Message}

We describe TEVAR in which the stent graft was deployed into the false lumen and rescued by a fenestration procedure called the "homing technique."

See Editorial Commentary page e45.

aorta through the LSA and one in the ascending aorta through the right femoral artery. Aortography confirmed

A 0.035-inch Lunderquist extra-stiff guidewire (Cook Medical Inc, Bloomington, Ind) was advanced into the ascending aorta. The stent graft (Valiant, 32/32 × 200 $\mathrm{mm}$, Medtronic Inc, Minneapolis, Minn) was deployed, covering half of the orifice of the LSA. Repeated angiography confirmed "sealing of the false lumen" without malperfusion of the visceral vessels. However, postprocedural CT angiography, performed 1 day later, showed that the distal part of the endograft was located in the false lumen of the descending aorta (Figure 1, $C$ and $D$ ). The patient's postoperative course was uneventful, except for mild back

It was thought that placing an additional stent may increase the risk of paraplegia; therefore, a rescue procedure was planned. However, this was performed 2 weeks later because of financial constraint and transfer of the patient to our center for further management. We planned to fenestrate the septum with an additional stent graft to direct the blood flow back into the true lumen. A 5F VERT catheter (Cordis Corp, Fremont, Calif) was placed into the true lumen under local anesthesia. An aortogram showed that the true lumen was compressed and had formed a blind 


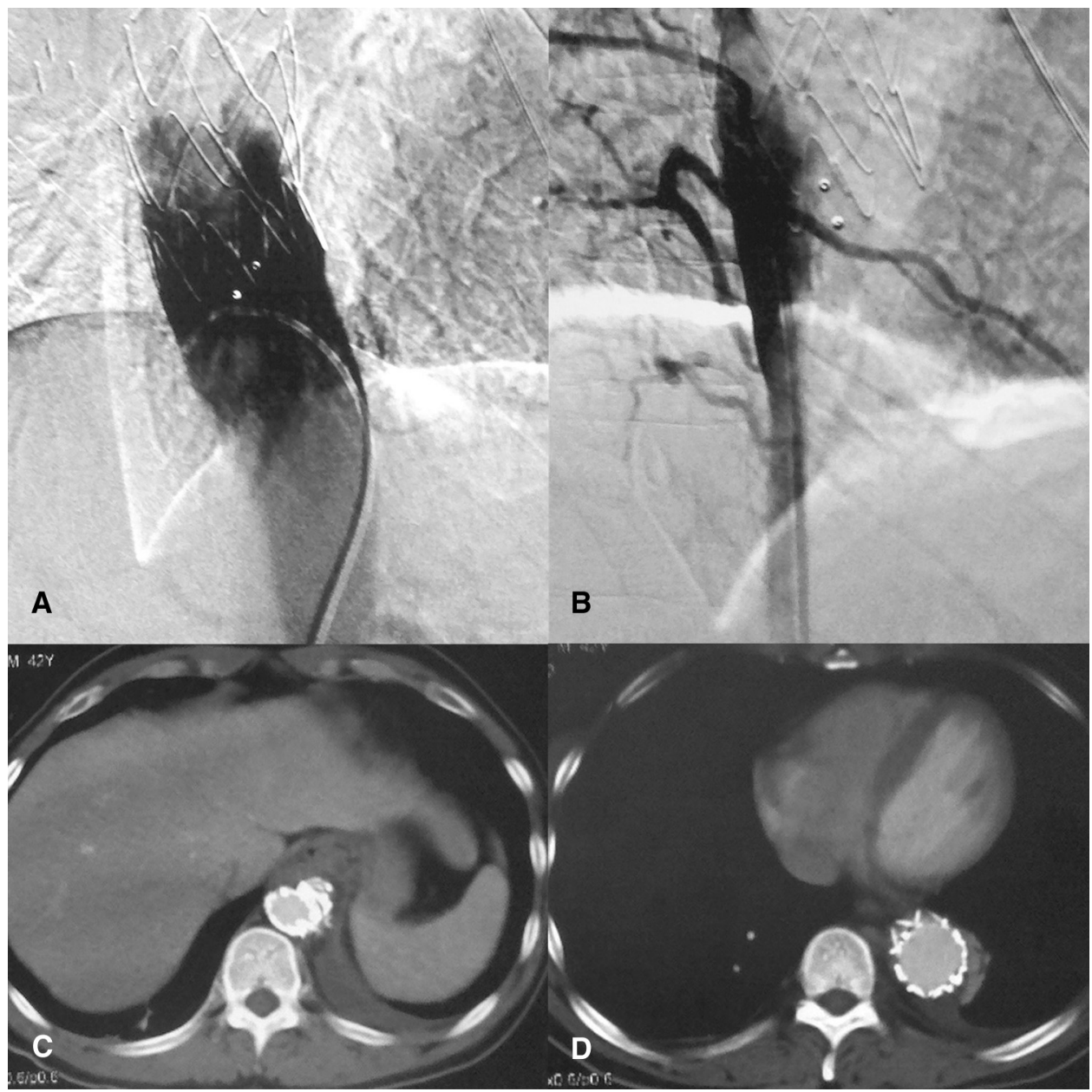

FIGURE 1. A and B, Aortography showing the stent graft connecting the true lumen and the false lumen. C and D, CT showing the distal part of the endograft located in the false lumen of the descending aorta.

tip at the middle of the stent graft, and that the false lumen was significantly dilated (Figure 1, $A$ and $B$ ). Although there was no endoleak around the stent graft site, there was a large distal tear found at the junction of the abdominal aorta and celiac trunk with outflow from the false lumen into the true lumen.

The proximal tip of the Lunderquist was advanced in a VERT catheter in an attempt to puncture the septum $20 \mathrm{~mm}$ below the previously deployed stent graft. However, several attempts to puncture the septum were unsuccessful even after using several different kinds of wires and catheters. Because prolonged procedural time under fluoroscopy may increase the risk of untoward complications, we stopped the attempts on fenestration.

The third procedure was carried out 1 week later after we received an Outback LTD Re-Entry Catheter (Cordis, a Johnson \& Johnson Co, Bridgewater, NJ). This is a specific intravascular device consisting of a $6 \mathrm{~F}$ compatible catheter with an angled needle that can penetrate the intima into the true lumen, which is usually used for lower-limb artery interventions. Under local anesthesia, a 10F Fustar steerable introducing sheath (LifeTech Co, Shenzhen, China) was placed in the true lumen of the descending aorta with the tip placed $20 \mathrm{~mm}$ below the previously deployed stent graft. The sheath was carefully positioned with its direction to the false lumen. The Outback LTD catheter was then advanced via the Fustar introducer with its tip against the septum (Figure 2, A). The needle was advanced to puncture the septum into the false lumen, guiding a wire into the stent graft and then to the ascending aorta (Figure 1, $B$ and $C$ ).

After gradually enlarging the hole using $8 \times 40$-mm and $14 \times 60$-mm balloons (Bard Co, Karlsruhe, Germany), a $24 \times 80$-mm bare stent (OptiMed Co, Ettlingen, Germany) was deployed in the true lumen for remodeling. Unfortunately, in the process of retrieving the delivery sheath, the stent was unintentionally pulled down below the renal abdominal aorta. Therefore, a $26 \times 80$-mm bare stent (OptiMed Co) was deployed (Figure 2, D and E). A $34 / 30 \times 160-\mathrm{mm}$ stent graft (LifeTech Co) was placed from the inner previous stent graft into the bare stent via the hole in the septum (Figure 2, F). 


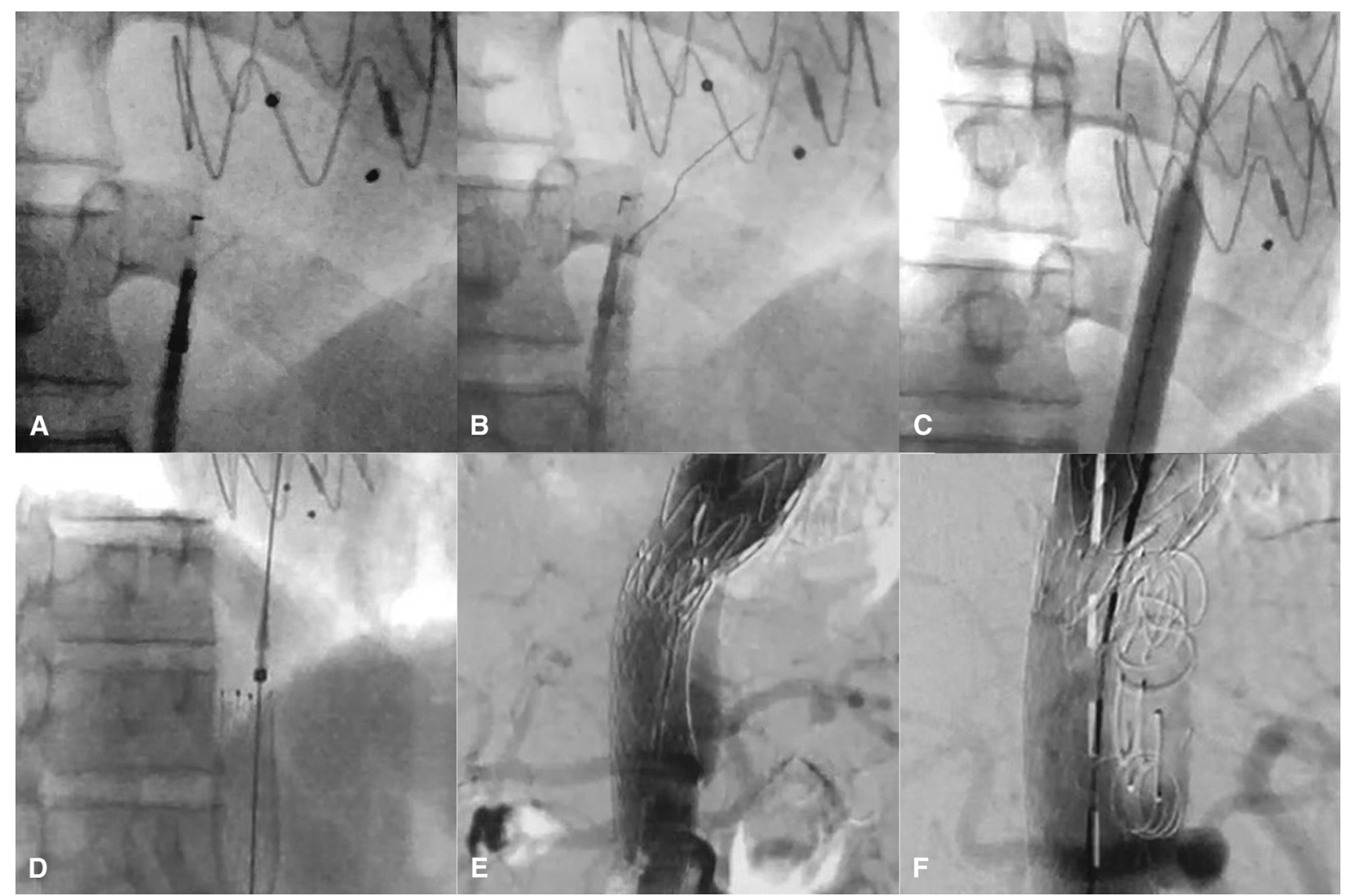

FIGURE 2. Successful endoluminal treatment of endovascular graft deployed in the false lumen. A, Adjusting outback direction and puncture. B, Guiding a wire into the stent graft after fenestration. C, Ballooning the fenestration. D, Bare stent remodeling. E, Additional stent graft placed after homing back with reflux. F, Coils placed into the false lumen.

The aortography showed that the blood flow was successfully drawn back into the true lumen without endoleak and aortic branches malperfusion, except the obvious reflux from the distal tear. Considering the short distance of the distal tear to the stent graft, 4 pairs of coils (Cook Medical Inc) with a diameter of $15 \mathrm{~mm}$ were placed into the false lumen near the distal tear via a VERT catheter. A repeated angiogram showed normal blood flow to the aorta, the major visceral arteries, and disappearance of the false lumen. The overall procedure time was 170 minutes, and estimated blood loss was approximately $350 \mathrm{~mL}$.

The patient's back pain disappeared the day after the procedure. No significant complications were observed. Aortic CT angiography 1 week postprocedure showed good positioning of the stent graft, bare stents, and coils, with normal blood flow to the aorta, patent visceral artery, and vanished false lumen (Figure 3). Eighteen months postprocedure, the false lumen completely disappeared without clinical or surgical complications.

\section{DISCUSSION}

Although medical management is still used in more than $60 \%$ of cases of type B aortic dissection, endovascular management (TEVAR) has increased from $7 \%$ to $31 \%$ in recent years. ${ }^{1}$ However, TEVAR is acknowledged as a promising and probably preferable method for patients with complicated Stanford type B aortic dissections. ${ }^{2}$ Its basic objective is to place a stent graft in the true lumen to cover the entry tear. However, a dissected and weakened vessel wall may rupture earlier after the stent graft has been unintentionally deployed into the false lumen. Previous reports of endograft deployment into the false lumen were described by Follisa and colleagues ${ }^{3}$ and Zhang and colleagues. ${ }^{4}$ In both of these studies, the guidewire was placed into the false lumen of the thoracic aorta arch during an attempt of TEVAR, similar to this case. Once the event has occurred, if the false lumen does not rupture, an emergency fenestration of the aorta distal to the endograft could reperfuse the true lumen. A second endograft should be implanted that connects the first endograft. In theory, this would be a reasonable approach but very ominous in practice because of the surgical challenge of treating the distal true lumen and visceral arteries. There are a few reports of this kind of procedure. ${ }^{3-5}$ As described in previous reports, surgical removal of the endoprosthesis is feasible but challenging. ${ }^{5,6}$ A hybrid operation to maintain blood supply of the visceral arteries would be necessary before 


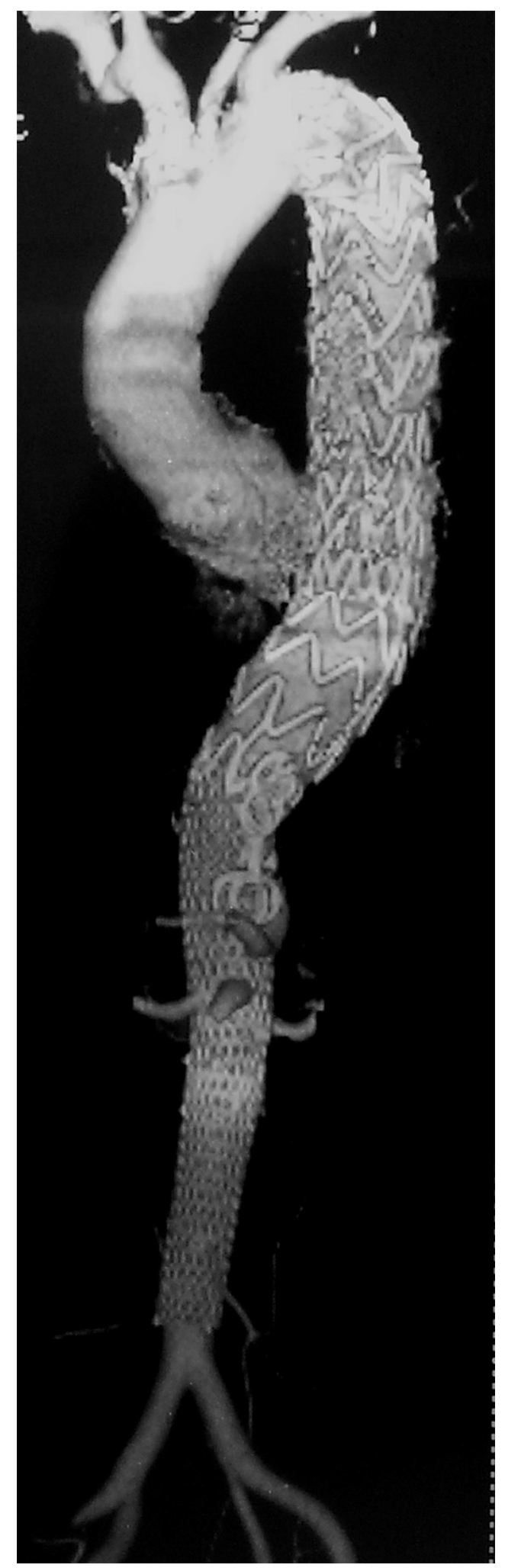

FIGURE 3. CT angiography showing good positioning of the stent graft and vanished false lumen 1 week postprocedure. the interventional treatment but potentially traumatic. That is why we chose the fenestration technique in this case.

In our case, the distal tear was so large that maintaining blood supply to the superior mesenteric artery and celiac trunk was of great concern. This gave us the opportunity to use the interventional method. Our strategy was to connect a newly placed stent graft into the previously deployed stent graft to draw the blood flow back into the true lumen, which can be called the "homing technique."

To fenestrate the aortic septum, the favorable distance to the previously placed stent graft may be 20 to $30 \mathrm{~mm}$ so that a long segment would be left for an additional stent graft without interfering with the blood supply to the visceral arteries.

Repeated attempts on fenestration were unsuccessful because the stiff wire failed to penetrate the membrane because of the narrow true lumen; thus, we chose the Outback LTD catheter for fenestration. It is a device with an L-shaped radiopaque marker at the catheter tip indicating needle direction, especially designed to reenter the true lumen in subintimal intervention of the lower leg arteries. ${ }^{7}$ In this case, we used a steerable introducing sheath to support the device and ensure a favorable direction and puncture. Although puncture of the descending aorta for accurate location and direction is still high risk, we found that using the Outback LTD device was useful for puncturing the septum.

As a general rule, a distal tear is left patent in most TEVAR procedures. ${ }^{8,9}$ However, in this case, the distal tear was very near to the stent graft and the reflux volume was large; therefore, we decided to seal the distal tear simultaneously. Because the location of the distal tear was just at the junction of the abdominal aorta and celiac trunk, necessitating a hybrid procedure, which is traumatic, we planned coil embolization as an additional procedure to TEVAR, expecting a "radical cure."10 Although the technique was not that difficult, we preferred to place a catheter before the bare stent that had been deployed in the aorta to prevent retro-migration of the coils. ${ }^{11}$ The proper positioning, control, and absolute certainty of the guidewire placement in the upstream process through the true lumen are crucial to the success of the procedure.

\section{CONCLUSIONS}

Despite the complications described earlier, TEVAR remains the therapy of choice for complicated type B aortic dissections. The technique of septum fenestration with the Outback catheter is effective and feasible by avoiding a traumatic hybrid procedure. With careful evaluation, mini-invasive interventional techniques should be applied to seal the distal tear in an attempt for "radical cure" of Stanford type B aortic dissection. 


\section{References}

1. Pape LA, Awais M, Woznicki EM, Suzuki T, Trimarchi S, Evangelista A, et al. Presentation, diagnosis, and outcomes of acute aortic dissection: 17-year trends from the International Registry of Acute Aortic Dissection. J Am Coll Cardiol. 2015;66:350-8

2. Criado FJ. Aortic dissection: a 250-year perspective. Tex Heart Inst J. 2011;38: 694-700.

3. Follisa F, Filipponea G, Stabileb A, Montalbanoa G, Florianod M, Finazzoc M, et al. Endovascular graft deployment in the false lumen of type B dissection. Interact Cardiovasc Thorac Surg. 2010;10:597-9.

4. Zhang R, Kofidis T, Baus S, Klima U. Iatrogenic type A dissection after attempted stenting of a descending aortic aneurysm. Ann Thorac Surg. 2006;82:1523-5.

5. Watanabe Y, Kuratani T, Shirakawa Y, Torikai K, Shimamura K, Sawa Y. Hybrid endovascular repair of a dissecting thoracoabdominal aortic aneurysm with stent graft implantation through the false lumen. J Vasc Surg. 2014;59:264-7.

6. Huang JH, Liu GQ, Wang W, Wang XW. Stent graft implantation through the false lumen in a Stanford B aortic dissection. Chin J Vasc Surg. 2010;2:244-6.
7. Husmann M, Federer J, Keo HH, Schmidli J, Kickuth R, Baumgartner I, et al. Bailout Revascularization of chronic femoral artery occlusions with the new Outback Catheter following failed conventional endovascular intervention. J Endovasc Ther. 2009;16:206-12.

8. Joung B, Ko YG, Park SH, Lee DY, Jang Y, Lim SH, et al. Expanding false lumen in the abdominal aorta 5 years after endovascular repair of a type B aortic dissection: successful exclusion of 3 distal re-entry sites. J Endovasc Ther. 2004;11: $577-81$.

9. Zhao J, Sai LK, Bin XH. Combined use of bare stent and coils in the treatment of aortic dissection with distal tear at celiac trunk. Natl Med China. 2012;92: 3333-6.

10. Kölbel T, Diener H, Larena-Avellaneda A, Debus S. Advanced endovascular techniques for thoracic and abdominal aortic dissections. J Cardiovasc Surg (Torino). 2013;54(1 Suppl 1):81-90.

11. Midulla M, Renaud A, Martinelli T, Koussa M, Mounier-Vehier C, Prat A, et al Endovascular fenestration in aortic dissection with acute malperfusion syndrome: immediate and late follow-up. J Thorac Cardiovasc Surg. 2011;142:66-72.

\title{
EDITORIAL COMMENTARY
}

\section{Endovascular rescue for complications during endovascular repair of type B dissection}

\author{
Stephen H. McKellar, MD, MSc
}

\footnotetext{
From the Division of Cardiothoracic Surgery, University of Utah School of Medicine, Salt Lake City, Utah. Disclosures: Author has nothing to disclose with regard to commercial support.

Received for publication Nov 3, 2015; accepted for publication Nov 4, 2015; available ahead of print Nov 25, 2015.

Address for reprints: Stephen H. McKellar, MD, MSc, Division of Cardiothoracic Surgery, University of Utah School of Medicine, 30 North 1900 East, 3C127 SOM, Salt Lake City, UT 84132 (E-mail: stephen. mckellar@hsc.utah.edu).

J Thorac Cardiovasc Surg 2016;151:e45-6

0022-5223/ $\$ 36.00$

Copyright (C) 2016 by The American Association for Thoracic Surgery

http://dx.doi.org/10.1016/j.jtcvs.2015.11.004
}

Acute aortic dissections are a challenging problem for cardiothoracic surgeons. Whereas traditionally an open repair has been needed, endovascular techniques are increasingly being used. In this issue of the Journal, Ma and colleagues ${ }^{1}$ report an endovascular approach to correcting blood flow after inadvertent deployment of a thoracic endovascular aortic repair stent graft in the false lumen. They report a multistage approach to solving this misadventure in an endovascular manner and provide some "how-to" technical advice about which catheters and endovascular devices they used in their successful fenestration of the aortic false lumen.

Ma and colleagues ${ }^{1}$ are to be congratulated for disclosing their intraoperative misadventures. Not only do they report the deployment of the device in the false lumen, they also report the stent graft migration that occurred during a later procedure. And herein lies the value of this report-how to get out of this problem endovascularly. Fortunately, it

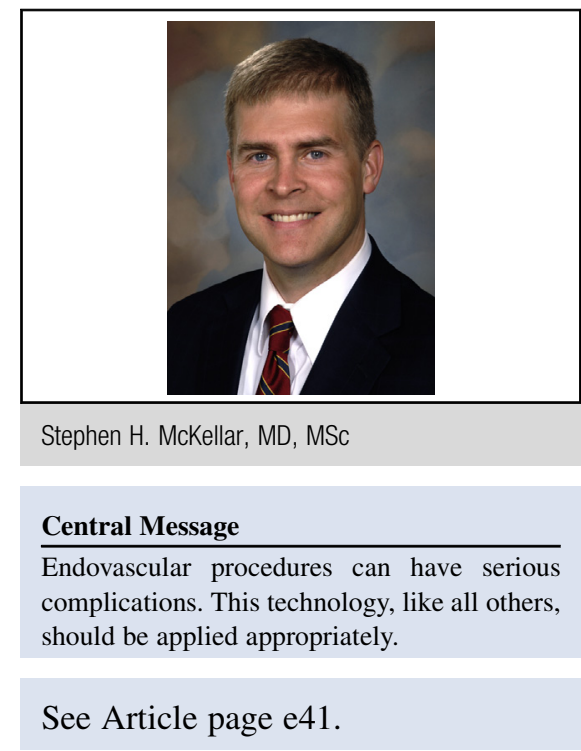

appears that the patient in the case report did well postoperatively.

This article has broader implications about the appropriate application of endovascular technologies of type $B$ dissections. Historically, the majority of patients with type B dissections have been managed nonoperatively with continued surveillance, with immediate operative intervention reserved for patients with symptoms, those 